\title{
Phase transitions in neutron stars and gravitational wave emission
}

\author{
G. F. Marranghello* and Cesar A. Z. Vasconcellos ${ }^{\dagger}$ \\ Instituto de Física, Universidade Federal do Rio Grande do Sul, Porto Alegre, Brazil \\ J. A. de Freitas Pacheco \\ Observatoire de la Côte d'Azur, Nice, France \\ (Received 24 June 2002; published 30 September 2002)
}

\begin{abstract}
We review the detectability of gravitational waves generated by oscillations excited during a phase transition from hadronic matter to deconfined quark-gluon matter in the core of a neutron star. Neutron star properties were computed using a Boguta-Bodmer based model and the MIT bag model. The maximum energy available to excite mechanical oscillations into the star is estimated by the energy difference between the configurations with and without a quark-gluon matter core. On basis of the planned sensitivity of present laser interferometers (VIRGO or LIGO I) and those of the next generation (LIGO II), the maximum volume to be probed by these experiments is determined. These results are used as an indication of the potential detectability of neutron stars as sources of gravitational waves. Our results indicate that the maximum distance probed by the detectors of the first generation is well beyond M31, whereas the second generation detectors will probably see phase transition events at distances two times longer, but certainly not yet attaining the Virgo cluster.
\end{abstract}

DOI: 10.1103/PhysRevD.66.064027

PACS number(s): 04.30.Db, 12.39.Ba, 21.60.-n, 26.60.+c

\section{INTRODUCTION}

The first generation of large gravitational interferometric detectors such as the French-Italian VIRGO and the American Laser Interferometric Gravitational Wave Observatory (LIGO), should be fully operational within one or two years. The best signal-to-noise $(\mathrm{S} / \mathrm{N})$ ratio that can be achieved from these detectors implies the use of matched-filter techniques that require a priori the knowledge of the signal waveform. Thus the identification of possible sources having a well-defined signal is a relevant problem in the detection strategy.

Neutron stars are certainly one of the most popular potential sources of gravitational waves (GWs), since they can emit by different mechanisms having a well-known waveform. Rotating neutron stars may have a time-varying quadrupole moment and hence radiate GWs, by either having a triaxial shape or a misalignment between the symmetry and the spin axes, which produces a precessional motion [1-3]. Moreover, fast rotating protoneutron stars may develop different instabilities such as the so-called ChandrasekharFriedman-Schutz (CFS) instability [4,5], responsible for the excitation of density waves traveling around the star in the sense opposite to its rotation, or undergo a transition from axisymmetric to triaxial shapes through the dynamical "barmode" instability [6]. All these mechanisms are potentially able to generate large amounts of energy in the form of GWs.

Nonradial oscillations are also a possible mechanism for neutron stars to emit GWs and this possibility was already discussed in the late 1960s [7]. One of the difficulties with this mechanism concerns the energy source necessary to excite the oscillations. The elastic energy stored in the crust and

\footnotetext{
*Electronic address: gfm@if.ufrgs.br

†Electronic address: cesarzen@if.ufrgs.br

†Electronic address: pacheco@obs-nice.fr
}

released by tectonic activity was recently considered [8], but the maximum available energy is likely to be of the order of $10^{44-45} \mathrm{erg}$. Thus even if all this energy could be converted into nonradial modes, the maximum distance that a signal could be seen by a laser interferometer like VIRGO is only about $3.0 \mathrm{kpc}$ [8]. This result depends, of course, on the adopted equation of state, which fixes the mode frequencies and damping time scales. A considerable amount of energy would be available if the neutron star undergoes a phase transition in the core. For instance, if quark deconfinement occurs, then the star will suffer a microcollapse since the equation of state of the quark matter is softer than that of the hadronic matter and the new equilibrium configuration will be more compact, having a larger binding energy. The energy difference will partially cover the cost of the phase transition and will partially be used to excite mechanical modes, whose energy will be dissipated by different channels. The physical conditions of matter at densities above the saturation density $\left(\rho_{0}=0.15 \mathrm{fm}^{-3}\right)$ is badly known and many first or second order phase transitions have been speculated upon. The possibility of a quark and hadron mixed phase was considered in Ref. [9], where the conservation of the electric and the baryonic charges are satisfied not locally but within a volume including many droplets of quarks and of hadrons. This mixed phase does not include local surface and Coulomb energies of quarks and nuclear matter and, as a consequence of these terms, if the interface tension between quark and nuclear matter is too large, the mixed phase is not favored energetically $[10,11]$. In this case, the neutron star will then have a core of pure quark matter and a mantle of hadrons surrounding it. In the absence of dynamical models able to follow the evolution of the star during the phase transition, we assume as in Ref. [12] that the structural rearrangement suffered by a "cold" star occurs on a dynamical time scale, i.e., of the order of milliseconds, shorter than the smooth global stellar readjustment suggested in Refs. $[13,14]$ and the gradual transition expected to occur in hot protoneutron stars 
[15]. Radial modes excited by the microcollapse do not radiate GWs, but an important coupling with rotation exists [16] and, if the star rotates, the oscillations will be damped not only by dissipation of the mechanical energy into heat but also by the emission of GWs.

In the present work, the detectability of GWs generated by oscillations excited during a phase transition in the core of a neutron star is reviewed [17]. Neutron star properties were computed using a description of the hadronic matter based on the work of Boguta and Bodmer [18], including the fundamental baryon octet, the isovector meson $\varrho$, and lepton degrees of freedom (see Ref. [15] for details). Hybrid models, including a quark-gluon core, with the same baryonic number as the pure hadron configuration were also computed and the energy difference between both states was used as an estimate of the maximum energy available to either excite mechanical oscillations or to be converted into heat. Then, using the planned sensitivity of present laser interferometers like VIRGO (or LIGO I) and those of the next generation (LIGO II), the maximum volume of space that can be probed by these experiments is calculated, since this indicates the potential detectability of these sources. The plan of this paper is the following: in Sec. II the star models are reviewed, in Sec. III the gravitational wave emission is discussed, and finally, in Sec. IV, conclusions are given.

\section{THE NEUTRON STAR MODEL}

The present model for the nuclear matter was already discussed by Marranghello et al. [19], who have investigated the effects of a finite temperature in the equation of state. Here, the same description for dense matter is adopted but only models with $T=0$ will be considered. Moreover, the coupling constants are slightly modified with respect to those considered in that work, in order to allow the maximum mass of the configuration to be compatible with recent results on the binary x-ray system Vela X-1 [20]. For the sake of completeness, we recall here the main points of the model.

The Lagrangian density describing the nuclear matter is

$$
\begin{aligned}
\mathcal{L}= & \sum_{B} \bar{\psi}_{B}\left[i \gamma_{\mu}\left(\partial^{\mu}-g_{\omega B} \omega^{\mu}\right)-\left(M_{B}-g_{\sigma B} \sigma\right)\right] \psi_{B} \\
& -\sum_{B} \bar{\psi}_{B}\left[\frac{1}{2} g_{\varrho B} \tau \cdot \varrho^{\mu}\right] \psi_{B}+\frac{b M}{3} \sigma^{3}+\frac{c}{4} \sigma^{4} \\
& +\frac{1}{2}\left(\partial_{\mu} \sigma \partial^{\mu} \sigma-m_{\sigma}^{2} \sigma^{2}\right)-\frac{1}{4} \omega_{\mu \nu} \omega^{\mu \nu}+\frac{1}{2} m_{\omega}^{2} \omega_{\mu} \omega^{\mu} \\
& -\frac{1}{4} \varrho_{\mu \nu} \cdot \varrho^{\mu \nu}+\frac{1}{2} m_{\varrho}^{2} \varrho_{\mu} \cdot \varrho^{\mu}+\sum_{l} \bar{\psi}_{l}\left[i \gamma_{\mu} \partial^{\mu}-M_{l}\right] \psi_{l} .
\end{aligned}
$$

TABLE I. Resulting coefficients.

\begin{tabular}{lcccc}
\hline \hline$\left(g_{\sigma} / m_{\sigma}\right)^{2}$ & $\left(g_{\omega} / m_{\omega}\right)^{2}$ & $\left(g_{\varrho} / m_{\varrho}\right)^{2}$ & $b(\times 100)$ & $c(\times 100)$ \\
\hline 9.927 & 4.820 & 4.791 & 0.8659 & -0.2421 \\
\hline \hline
\end{tabular}

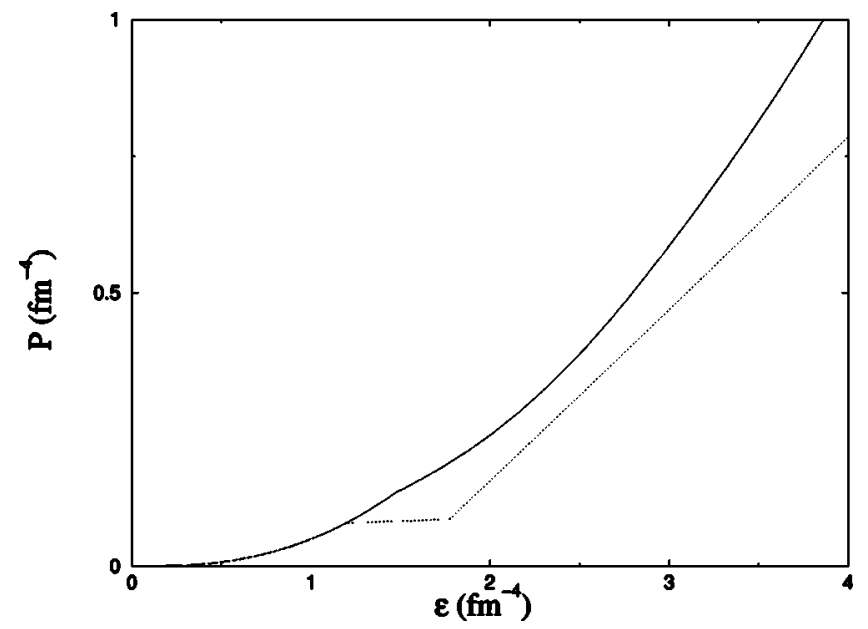

FIG. 1. Equation of state for hadronic matter (solid line) and for the quark-gluon matter (dotted line).

This equation represents nuclear matter as composed by a mixture of the fundamental baryon octet $\left(p, n, \Lambda, \Sigma^{+}, \Sigma^{0}, \Sigma^{-}, \Xi^{-}, \Xi^{0}\right)$ coupled to three mesons $(\sigma, \omega, \varrho)$ and leptons (for the details see [15]). The scalar and vector coupling constants, $g_{\sigma}, g_{\omega}$ and the coefficients $b$, $c$ were determined by imposing that the model bulk properties should be able to reproduce the binding energy $E_{b}$ (= $-16.3 \mathrm{MeV})$, the compression modulus $K(=240 \mathrm{MeV})$, and the nucleon effective mass $M^{*}=M-g_{\sigma} \bar{\sigma}$ $(=732 \mathrm{MeV})$ at the saturation density $\rho_{0}\left(=0.153 \mathrm{fm}^{-3}\right)$. Additionally, the isovector coupling constant $g_{\varrho}$ is determined from the coefficient for the symmetry energy in nuclear matter, $a_{4}(=32.5 \mathrm{MeV})$. We have used the universal hyperon-nucleon coupling ratios $\chi_{i}=g_{H i} / g_{i}$, with $i$ $=\sigma, \varrho, \omega$. The resulting coefficients used in our computations are given in Table I. Figure 1 shows the equation of state derived from our model and shown in Fig. 2 is the energy density profile inside the star for two configurations having gravitational masses equal to 1.2 and $1.6 M_{\odot}$ respectively.

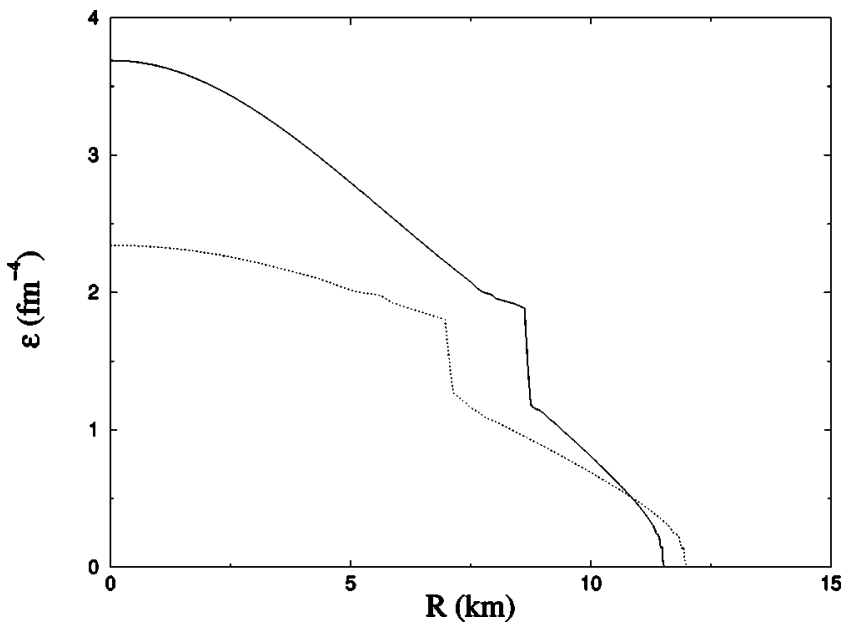

FIG. 2. Neutron star energy density-radius relation for $M$ $=1.6 M_{\odot}($ solid line $)$ and $M=1.2 M_{\odot}($ dotted line $)$ on which the quark-gluon core extends up to 8.6 and $7 \mathrm{~km}$, respectively. 
TABLE II. Physical conditions at the phase transition: Energy densities and pressure are given in $\mathrm{GeV} \mathrm{fm}^{-3}$.

\begin{tabular}{ccc}
\hline \hline$\epsilon_{H}$ & $\epsilon_{q}$ & $P$ \\
\hline 0.236 & 0.349 & 0.0163 \\
\hline \hline
\end{tabular}

Hybrid models including a quark-gluon core were also computed. The quark matter was described by the MIT Lagrangian [21] and the physical conditions at the deconfinement transition were estimated from the Gibbs criteria, namely, by the equality of the chemical potential and pressure of both phases, under conservation of the baryon number and electrical charge. The physical parameters at the transition point are given in Table II, for a bag constant $B^{1 / 4}=180 \mathrm{MeV}$ and a strange quark mass $m_{s}=150 \mathrm{MeV}$. The gravitational mass of the star as a function of the central energy density is shown in Fig. 3 for the case of a pure hadronic configuration (dashed line), a hybrid star with a quark core (solid line), and for comparison, a case where a mixed phase exists in the center, computed in the same way as Ref. [9].

It should be emphasized that our equation of state is quite steep ( $d \log P / d \log \varrho \approx 2.6$ ) near saturation and, as a consequence, the deconfinement transition occurs at densities just above the saturation value $\left(\rho \sim 1.7 \rho_{0}\right)$, producing hybrid stars with very extended quark-gluon cores. Here we take the opportunity to reiterate that, from the actual status of theoretical predictions to the EOS, with so many parameters to be adjusted, even in the quark phase and specially in the hadron phase, we believe that only qualitative results can be obtained with some insights on the quantitative results.

The maximum stable mass of a pure hadronic configuration is about $M_{\max } \approx 2.1 M_{\odot}$ while for hybrid stars this limit is reduced to $1.73 M_{\odot}$. Thus the present calculations exclude the possibility that Vela X-1 has a quark core. Notice that our models obey the Seidov criterium [22], namely, that the hybrid star will be stable only if the energy jump across the

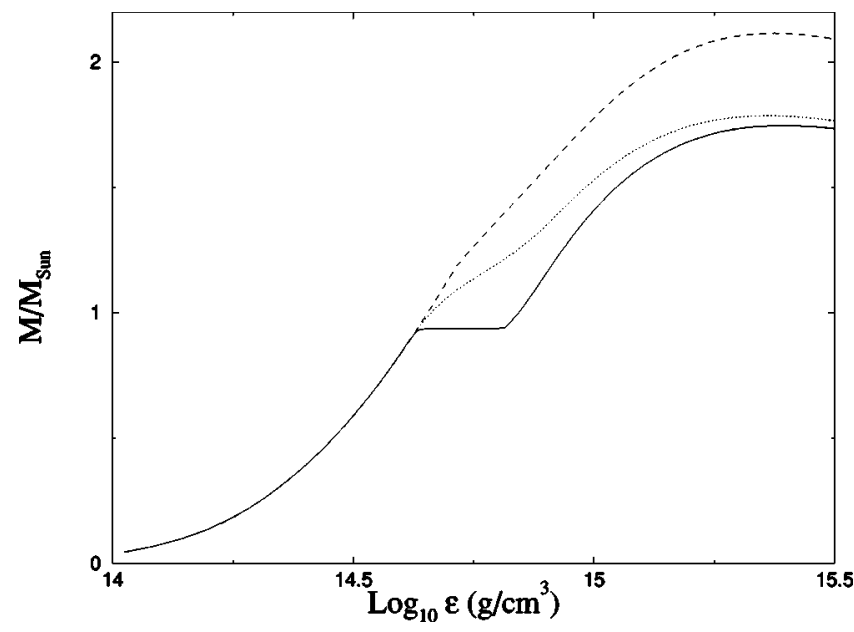

FIG. 3. Neutron star mass as a function of central energy density for a hybrid star with constant pressure transition (solid line), hybrid (dotted line), and pure hadronic star (dashed line) configurations. transition surface satisfies the condition

$$
\frac{\epsilon_{q}}{\epsilon_{H}}<\frac{3}{2}\left(1+\frac{P}{\epsilon_{H}}\right)
$$

where $P, \epsilon_{q}, \epsilon_{H}$ are, respectively, the pressure, and the energy density of quarks and hadrons at the transition point.

One of the goals of this work is the determination of an upper limit for the energy able to excite the different oscillation modes of the star, when a modification in its internal structure occurs. In this sense, the simplest approach is to compute the energy difference between two configurations having the same baryonic number; the first constituted of pure hadrons $(\mathrm{H})$, the second having a core of deconfined matter (HQ), and use such a difference as an indication of the maximum available energy. Table III gives the parameters for five models defined by a given baryonic number [23]

$$
N=\int_{0}^{R} 4 \pi r^{2}\left[1-\frac{2 G m(r)}{r^{2}}\right]^{-1 / 2} \rho_{B}(r) d r,
$$

or the baryonic mass of the star $\left(M_{b a r}=N M_{B}\right)$; the expected gravitational mass of both configurations (pure hadron and hybrid),

$$
M_{g}=m(R)=\int_{0}^{R} 4 \pi r^{2} \epsilon(r) d r
$$

expected radii, masses of the quark-gluon core, and the energy difference obtained from the passage of configuration $\mathrm{H}$ to $\mathrm{HQ}$, the binding energy.

$$
E_{g}=M_{g}-M_{b a r}
$$

\section{THE GRAVITATIONAL WAVE EMISSION}

The transition from configuration $\mathrm{H}$ to $\mathrm{HQ}$ may occur through the formation of a mestastable core, built-up by an increasing central density. The increase in the central density may be a consequence of a continuous spin-down or other different mechanisms the star could suffer. This transition releases energy, exciting mainly the radial modes of the star [17]. These modes do not emit GWs, unless when coupled with rotation [16], a situation which will be assumed here.

In order to simplify our analysis, we will consider that most of the mechanical energy is in the fundamental model. In this case, the gravitational strain amplitude can be written as

$$
h(t)=h_{0} e^{-\left(t / \tau_{g w}-i \omega_{0} t\right)}
$$

where $h_{0}$ is the initial amplitude, $\omega_{0}$ is the angular frequency of the mode, and $\tau_{g w}$ is the corresponding damping time scale. The initial amplitude is related to the total energy $E_{g}$ dissipated under the form of GWs by the relation [24]

$$
h_{0}=\frac{4}{\omega_{0} r}\left[\frac{G E_{g}}{\tau_{g w} c^{3}}\right]^{1 / 2},
$$


TABLE III. Parameters of the stellar models: Masses are in solar units and radii in $\mathrm{km} ; \mathrm{H}$ and HQ mean pure hadron and hybrid configurations, respectively.

\begin{tabular}{rcccccc}
\hline \hline$M_{\text {bar }}$ & $M_{g}(\mathrm{H})$ & $M_{g}(\mathrm{HQ})$ & $M_{g}($ core $)$ & $R(\mathrm{H})$ & $R(\mathrm{HQ})$ & $\log (E) \operatorname{erg}$ \\
\hline 1.0749 & 1.0003 & 0.9625 & 0.1150 & 12.82 & 12.47 & 52.83 \\
1.3202 & 1.2009 & 1.0953 & 0.3608 & 13.04 & 12.16 & 53.28 \\
1.5724 & 1.4008 & 1.2468 & 0.6013 & 13.13 & 11.98 & 53.44 \\
1.8342 & 1.6006 & 1.4164 & 0.8626 & 13.01 & 11.82 & 53.52 \\
2.1045 & 1.8002 & 1.6141 & 1.1766 & 12.69 & 11.50 & 53.52 \\
\hline \hline
\end{tabular}

where $G$ is the gravitational constant, $c$ is the velocity of light, and $r$ is the distance to the source.

Relativistic calculations of radial oscillations of a neutron star with a quark core were recently performed by Sahu et al. [25]. However, the relativistic models computed by those authors do not have a surface of discontinuity where an energy jump occurs. Instead a mixing region was considered, where the charges (electric and baryonic) are conserved globally but not locally [9]. Oscillations of star models including an abrupt transition between the mantle and the core were considered by Haensel [26] and Miniutti [27]. However, a Newtonian treatment was adopted and the equation of state used in the calculations does not correspond to any specific nuclear interaction model. In spite of these simplifications, these hybrid models suggest that rapid phase transitions, such as that resulting from the formation of a pion condensate, proceed at the rate of strong interactions and affect substantially the mode frequencies. However, the situation is quite different for slow phase transitions (the present case), where the mode frequencies are quite similar to those of a one-phase star [26]. In this case, scaling the results of [26], the frequency of the fundamental mode (uncorrected for gravitational redshift) is given approximately by

$$
\nu_{0} \approx 63.8\left[\frac{\left(M / M_{\odot}\right)}{R^{3}}\right]^{1 / 2} \mathrm{kHz},
$$

where the mass is given in solar units and the radius in $\mathrm{km}$.

Once the transition to quark-gluon matter occurs, the weak interaction processes for the quarks $u, d$, and $s$

$$
u+s \rightarrow d+u
$$

and

$$
d+u \rightarrow u+s
$$

will take place. Since these reactions are relatively slow, they are not balanced while the oscillations last and thus, they dissipate mechanical energy into heat [28]. According to calculations of Ref. [21], the dissipation time scale can be estimated by the relation

$$
\tau_{d} \approx 0.01\left(\frac{150 \mathrm{MeV}}{m_{s}}\right)^{4}\left(\frac{M_{\odot}}{M_{c}}\right) s,
$$

where $m_{s}$ is the mass of the $s$-quark in $\mathrm{MeV}$ and $M_{c}$ is the mass of the deconfined core in solar masses. This equation is valid for temperatures in the range $10^{8}-10^{9} \mathrm{~K}$. On the other hand, according to Ref. [16] the damping time scale by GW emission is

$$
\tau_{g w}=1.8\left(\frac{M_{\odot}}{M}\right)\left(\frac{P_{m s}^{4}}{R^{2}}\right) s,
$$

where again the stellar mass is in solar units, the radius is in $\mathrm{km}$, and the rotation period $P$ is in milliseconds.

In a first approximation, the fraction of the mechanical energy which will be dissipated under the form of GWs is $f_{g}=1 /\left(1+\tau_{g w} / \tau_{d}\right)$. Notice that the damping time scale by $\mathrm{GW}$ emission depends strongly on the rotation period. Therefore one should expect that slow rotators will dissipate most of the mechanical energy into heat. In Table IV is given for each star model the expected frequency of the fundamental mode (corrected for the gravitational redshift), the critical rotation period (in ms) for having $f_{g}=0.50$, the GW damping for this critical period, and the quality factor of the oscillation, $Q=\pi \nu_{0} \tau_{g w}$.

After filtering the signal, the expected signal-to-noise ratio is

$$
(S / N)^{2}=4 \int_{0}^{\infty} \frac{|\widetilde{h}(\nu)|}{S_{n}(\nu)} d \nu
$$

where $\widetilde{h}(\nu)$ is the Fourier transform of the signal and $\mathrm{S}_{n}(\nu)$ is the noise power spectrum of the detector. Performing the required calculations, the $\mathrm{S} / \mathrm{N}$ ratio can be written as

$$
(S / N)^{2}=\frac{4}{5} h_{0}^{2}\left(\frac{\tau_{g w}}{S_{n}\left(\nu_{g w}\right)}\right) \frac{Q^{2}}{1+4 Q^{2}} .
$$

TABLE IV. Oscillation parameters: The damping time scale $\tau_{g w}$ is given for the critical period; maximum distances for VIRGO (V) and LIGO II (L) are in Mpc.

\begin{tabular}{cccccc}
\hline \hline $\begin{array}{c}\nu_{0} \\
(\mathrm{kHz})\end{array}$ & $\begin{array}{c}P_{\text {crit }} \\
(\mathrm{ms})\end{array}$ & $\begin{array}{c}\tau_{g w} \\
(\mathrm{~ms})\end{array}$ & $Q$ & $\begin{array}{c}D_{\max } \\
(\mathrm{VIRGO})\end{array}$ & $\begin{array}{c}D_{\max } \\
(\mathrm{LIGO} \text { II })\end{array}$ \\
\hline 1.62 & 1.64 & 87.0 & 442 & 4.9 & 10.2 \\
1.83 & 1.25 & 27.0 & 155 & 6.4 & 13.5 \\
2.06 & 1.13 & 17.0 & 110 & 6.0 & 12.8 \\
2.32 & 1.06 & 11.5 & 84 & 5.1 & 11.1 \\
2.72 & 1.00 & 8.4 & 72 & 3.6 & 5.7 \\
\hline \hline
\end{tabular}


In the equation above, the angle average on the beam factors of the detector were already performed [29].

From Eqs. (4) and (11), once the energy and the $S / N$ ratio are fixed, one can estimate the maximum distance $D_{\max }$ to the source probed by the detector. In the last two columns of Table IV are given distances $D_{\max }$ derived for a signal-tonoise ratio $\mathrm{S} / \mathrm{N}=2.0$ and the sensitivity curve of the laser beam interferometers VIRGO and LIGO II. In both cases, it was assumed that neutron stars underwent the transition having a rotation period equal to the critical value.

We emphasize again that our calculations are based on the assumption that the deconfinement transition occurs in a dynamical time scale [12]. In the scenario developed in Ref. [13], a mixed quark-hadron phase appears and the complete deconfinement of the core occurs according to a sequence of quasiequilibrium states. The star contracts slowly, decreasing its inertia moment and increasing its angular velocity until the final state can be reached in a time scale of the order of $10^{5} \mathrm{yr}$ [13]. Clearly, in this scenario no gravitational waves will be emitted and this could be a possibility to discriminate both evolutionary paths.

\section{CONCLUSIONS}

The structure of pure hadronic configurations and that of hybrid stars having a quark core, with the same baryonic number, were computed using a new equation of state [19]. The maximum stable mass for pure hadronic configurations satisfies the requirement of being higher than the mass of Vela X-1 $\left(M=1.86 \pm 0.16 M_{\odot}\right)$, determined recently [20] from a new study of its orbital motion.

As one should expect, the masses of quark cores increase

[1] A. Ferrari and R. Ruffini, Astrophys. J. 158, L71 (1969).

[2] M. Zimmerman and E. Szedenits, Phys. Rev. D 20, 351 (1979).

[3] S. Shapiro and S. Teukolsky, Black Holes, White Dwarfs and Neutron Stars (Wiley, New York, 1983).

[4] S. Chandrasekhar, Phys. Rev. Lett. 24, 611 (1970).

[5] J. Friedman and B. Schutz, Astrophys. J. 222, 281 (1978).

[6] D. Lai and S. Shapiro, Astrophys. J. 442, 259 (1995).

[7] K. Thorne and A. Campolattaro, Astrophys. J. 149, 591 (1967).

[8] J. de Freitas Pacheco, Astron. Astrophys. 336, 397 (1998).

[9] N. Glendenning, Phys. Rev. D 46, 1274 (1992).

[10] H. Heiselberg, C.J. Pethick, and E.F. Staubo, Phys. Rev. Lett. 70, 1355 (1993).

[11] H. Heiselberg and M. Hjorth-Jensen, Phys. Rev. Lett. 80, 5485 (1999).

[12] P. Haensel and M. Prózynski, Astrophys. J. 258, 306 (1982).

[13] N. Glendenning, S. Pei, and F. Weber, Phys. Rev. Lett. 79, 1603 (1997).

[14] N. Glendenning and F. Weber, Astrophys. J. Lett. 559, L119 (2001).

[15] T. Baumgarte, H.-T. Janka, W. Keil, S. Shapiro, and S. Teukolsky, Astrophys. J. 468, 823 (1996).

[16] W.-Y. Chau, Astrophys. J. 147, 664 (1967). with the baryon number of the configuration, as well as the difference between the binding energies between hybrid and single phase objects. On the basis of this result and from the point of view of the GW emission, it would be natural to expect that phase transitions occurring in more massive stars would be more easy to detect. In fact, this is not the case because on the one hand the damping time scale due to the emission of GWs is inversely proportional to the mass and hence massive stars have low oscillation quality factors and, on the other hand, the detectors have sensitivities optimized at frequencies around $200 \mathrm{~Hz}$. In comparison with nonradial oscillations (for instance $m=l=2$ modes), these have comparable frequencies but damping time scales one order of magnitude higher than those resulting from radial modes coupled to rotation. In this case, higher quality factors $Q$ may be obtained but most of the mechanical energy will be dissipated into heat. In order to be an efficient source of GWs by the mechanism considered here, the star must be a fast rotator, i.e., to have rotation periods of the order of a few milliseconds. Even in the most favorable case (model 2, corresponding to a baryonic mass equal to $\left.1.32 M_{\odot}\right)$, if the rotation period is $4.0 \mathrm{~ms}$, only $1 \%$ of the available energy will be emitted as GWs.

Inspection of Table IV indicates that the maximum distance probed by detectors of first generation (VIRGO, LIGO I) is about $6.4 \mathrm{Mpc}$, well beyond M31, whereas the second generation (LIGO II) will probably see phase transition events at distances two times longer, but certainly not yet attaining the Virgo cluster. The small probed volume and the rapid rotation required for this mechanism to be efficient imply a low event rate, imposing severe limitations on the detectability of such a signal.

[17] H. Sotani, K. Tominaga, and K. Maeda, Phys. Rev. D 65, 024010 (2002).

[18] J. Boguta and A. Bodmer, Nucl. Phys. A292, 413 (1977).

[19] G. Marranghello, C.A.Z. Vasconcellos, M. Dillig, and J.A. de Freitas Pacheco, Int. J. Mod. Phys. E 11, 83 (2002).

[20] O. Barziv, L. Kaper, M. van Kerkwijk, J. Telting, and J. van Paradijs, Astron. Astrophys. 377, 925 (2001).

[21] T. de Grand, R. Jaffe, K. Johnson, and J. Kiskis, Phys. Rev. D 12, 2060 (1975).

[22] Z. Seidov, Astron. Zh. 48, 443 (1971).

[23] S. Weinberg, Gravitation and Cosmology (Wiley, New York, 1972).

[24] J. de Freitas Pacheco, VI International Workshop on Relativistic Aspects of Nuclear Physics, edited by T. Kodama et al. (World Scientific, Singapore, 2001), p. 158.

[25] P. Sahu, G. Burgio, and M. Baldo, Astrophys. J. Lett. 566, L89 (2002).

[26] P. Haensel, J. Zdunik, and R. Schaeffer, Astron. Astrophys. 217, 137 (1989).

[27] G. Miniutti, J.A. Pons, E. Berti, L. Gualtieri, and V. Ferrari, astro-ph/0206142.

[28] Q. de Wang and T. Lu, Chin. Astron. Astrophys. 9, 159 (1984).

[29] T. Regimbau, Ph.D. thesis, University of Nice, 2001. 